\title{
Mito della crisi e crisi del mito in Aracoeli di Elsa Morante
}

\author{
Angela DI FAZIO ${ }^{1}$ \\ Università degli Studi di Bologna \\ angela.difazio3@unibo.it
}

Recibido: $15 / 12 / 2012$

Aceptado: 20/06/2013

\section{RIASSUNTO}

Lo studio verifica la presenza di un ipotesto antropologico nel romanzo di Morante, a partire da un nucleo analitico di impostazione metodologica attorno a una casistica di diagnosi psicopatologica, a sua volta sorgiva delle teorie demartiniane sulla crisi della cultura e sul suo conseguente riassorbimento mediante operazioni rituali, quali la letteratura mostra di essere nella fattispecie del racconto e della simbologia mitologica.

Parole chiave: apocalisse, fenomenologia della crisi, De Martino, mito, psicologia primitiva.

\section{The Myth of Crisis and the Crisis of Myth in Morante's Aracoeli}

\begin{abstract}
The study proves the presence of an anthropological hypotext in Morante's novel, methodologically approached through a series of psychopathological diagnoses that followed De Martino's theory on the crisis of culture and its subsequent reassumption by ritual operations, where Literature shows to be in the case of the story and mythological symbolism.
\end{abstract}

Keywords: Apocalypse, Phenomenology about Crisis, De Martino, Myth, Primitive Psychology.

Il mito dell'apocalisse ${ }^{2}$, che informa di sé il romanzo Aracoeli, ha una precisa rispondenza entro il complesso unitario originato dalla morantiana «scienza del

${ }^{1}$ Università degli Studi di Bologna, Dipartimento di Filologia Classica e Italianistica, via Zamboni 32, I-40100, Bologna (Bo), Italia.

${ }^{2}$ Se l'«aspetto apocalittico» del romanzo è già rilevato da Rosa (Rosa 2006: 300), più propriamente di «visione» apocalittica parla Bazzocchi (2009: 144), come pure Pischedda (2004) che non a caso inserisce una riflessione critica sul romanzo morantiano. Ma la componente «disforica» del testo è rilevata pure da Mengaldo (1994) e Paduano (1994). 
mito» in accordo con la finzionale «esperienza del mito» ${ }^{3}$, propria di ciascun personaggio attivo nell'opera di Morante. Solo supponendo una dinamica dialettica tra la componente mitopoietica delle figure agenti nel testo e l'analisi critica autoriale, che ad esse si appunta con intenti smaganti, è possibile infatti collocarci oltre il cliché dell'irrazionalismo morantiano, per valutare come la stessa struttura del pensiero mitico, se oggetto di «genuina» ${ }^{4}$ riflessione, arrivi «a contrapporsi all'inconscio come una barriera, escludendone un predominio indiscriminato» (Jesi 1992: 36). A maggior ragione, se si consideri che persino una puntuale scrittura storica, ovvero la descrizione di una determinata epoca, non può prescindere dalla realizzazione, dalla «prestazione linguistica» (Koselleck 2009: 13), che spazi «dal mito al romanzo», dell'epoca in questione.

La Morante, storica e mitografa, trascrittrice sulla carta di miti impersonati, appronta una documentazione scientifica di valore quasi museografico sulla cosiddetta «civiltà contadina», perifrasi per una più generica componente popolare, ma anche, appunto, sulla realtà del sottoproletariato urbano, ad essa assimilata pasolinianamente nel segno arbitrario della pre-storicità, persino nella variante del mondo operaio (vedasi, a questo proposito, la "queste industriale" di Davide Segre ne La Storia). Sono, allora, i personaggi creati da Morante a dare ricetto all'epifania mitica, di cui si fanno veicolo simbolico. Si può concludere che, nel concerto delle valutazioni autoriali, vada discusso non di questione ideologica, con quello che di

${ }^{3}$ La divaricazione tra le due categorie modali è introdotta assai perspicuamente da Furio Jesi a proposito della sensibilità mitica, che organizza il pensiero umanistico-rinascimentale: «[...] il primo umanesimo e la cultura del rinascimento furono sostanzialmente estranei alla 'scienza del mito'. Essi 'vissero', o cedettero di vivere il mito, con la predilezione simpatetica ben più che scientifica [...]. Occorre qui distinguere fra esperienza del mito e scienza del mito, ché ambedue non sono necessariamente compagne (anche se, mentre l'esperienza del mito può senza danno far a meno della scienza, la 'scienza del mito' priva dell'esperienza di esso è davvero assai fragile)» (Jesi 1989: 28-30). Jesi individua un'eccezione alla norma rinascimentale nella speculazione di Pico della Mirandola, che, attivo conoscitore della tradizione cabalistica, avrebbe trovato nel misticismo ebraico il puntello per la ricerca sistematica sulla verità essenziale del mito, alla base di una scienza ad esso correlata. Non diversamente, fatta salva la distanza cronologica, si potrebbe argomentare a proposito di una Morante in sintonia col concetto ebraico di «esilio» da una condizione di sapere e felicità primordiali. Che poi, nel pensiero cabalistico, la tematica dell'esilio sia direttamente associata all'apocalittica è attestato da Scholem, secondo cui, a partire dalla cacciata spagnola del 1492, per l'ebraismo messianico, «I giorni della fine e la via per giungere ad essi divennero così importanti come i primi: più che risalire ai primordi della storia o alle sue premesse metafisiche aveva valore adesso conquistare lo stadio finale del processo cosmologico. [...] l'apocalittica - l'evocazione di tutte le forze capaci di sollecitare e provocare la crisi della 'fine' - diventa la meta dei mistici» (Scholem 1993: 259).

${ }^{4}$ La contrapposizione di valore tra «mito genuino» e «mito tecnicizzato» - ricorda Jesi è resa funzionale da Kerényi in risposta alla strumentalizzazione politica del mito da parte della «destra tradizionale» (Jesi 1989: 78-81). 
reazionario gli intellettuali del dopoguerra seppero individuare nella speculazione mitologica, filofascista nei suoi estremi ${ }^{5}$, quanto di una precisa scelta metodologica di calco plastico, anche se non neutrale, di quella condizione materiale e psichica descritta dall'antropologo De Martino ne Il mondo magico.

Nel particolare umanesimo morantiano, non lontano - a nostro avviso - da quello «etnografico» dello studioso napoletano, il mito, come narrazione rituale, rientrerebbe nel processo di «creazione di forme culturali definite», ossia nel «complesso di esperienze e di rappresentazioni, di misure protettive e di pratiche», che contrastano il «rischio esistenziale» di presenze individuali inermi e soccombenti di fronte alle difficoltà del vivere quotidiano, e che, per queste stesse presenze in crisi, costituiscono una forma primaria di «riscatto culturale» (De Martino 1997: 74-75). Di qui, l'accezione del concetto di «crisi» come «movimento» e «sviluppo» - con osmosi dei due piani di spazialità (reale e figurata): esterno/interno -, accezione ${ }^{6}$ da tener presente nel considerare, fondamentalmente $\mathrm{e}$ in linea con le indicazioni critiche precedenti, il romanzo Aracoeli in quanto racconto di viaggio. Ponendo come premessa, pur ai limiti del tautologico, il carattere biografico di un romanzo eponimo, quale si evince dall'epigrafico incipit («Mia madre era andalusa. [...] Di suo nome di battesimo, si chiamava Aracoeli», Morante 1982: 3), o per essere più esatti postulando la presenza di un biografismo di secondo grado - corrispondente al piano dell' «anamnesi postuma» circa la vicenda aracoeliana -, inserito nel contesto del narrato biografico e autofinzionale, quello delle «rimembranze apocrife» ${ }^{7}$ (Morante 1982: 13), di Manuele, soffermiamoci sul motivo del viaggio come reagente della narrazione:

E su di me la decisione di questa partenza dirompeva in un sentimento estremo di rischio e di follia; ma anche di un ignoto entusiasmo (enthusiasmòs = invasione divina). [...] l'enthusiasmòs m'ha insegnato l'unico itinerario a me possibile: comandato, anzi. [...] E così, adesso (l'ora è circa le undici di mattina) mi sono messo sulla strada, in partenza da

${ }^{5}$ Si notino, a questo riguardo, le recise prese di distanza di De Martino dall'operazione editoriale, condotta in collaborazione con Cesare Pavese, della «Collana viola» einaudiana, ritenuta dallo studioso «estremamente pericolosa, perché in essa si riflette, con particolare evidenza, la crisi della cultura borghese, le sue contraddizioni e le sue ultime alcinesche seduzioni $[. .$.$] il carattere di una torbida evasione irrazionalistica, di sfiducia della ragione,$ di idoleggia mento acritico dell'arcaico secondo l'impulso di un romanticismo fuor di stagione» (Pavese, De Martino 1991: 181-203).

${ }_{6}^{6}$ Si noti come tale definizione della crisi ne faccia un «concetto periodale iterativo», ossia la determina come una situazione di «oltrepassa mento di una soglia epocale» (Koselleck 2009: 100) con espliciti richiami al contesto teologico del pensiero apocalittico, per cui la crisi corrisponderebbe a «un'epoca di transizione dominata dalla Fine» (Kermode 2004: 16) con le conseguenti ricadute sul piano della concezione della storia.

${ }^{7}$ Le definizioni di «anamnesi postuma» e di «rimembranze apocrife», che assumiamo da Morante, ci sembrano contraddistinguere e sintetizzare i due aspetti fondamentali della narrazione. 
Milano, per andare alla ricerca di mia madre Aracoeli nella doppia direzione del passato e dello spazio. (Morante 1982: 8-9)

Un indicatore contestuale è qui l'utilizzo dei termini «rischio» e «follia», benché seguiti dal più attrattivo e rilevato «entusiasmo», che di-verte il lettore, appena edotto da Manuele sulla sua attività di traduttore di pubblicazioni a carattere divulgativo, attività a cui il personaggio narrante sembra ora ammiccare, più o meno in buona fede, c'est à dire autoironicamente, nel creare un campo semantico che rimandi alla sacertà. Ma la componente di perniciosità del viaggio, come esperienza psicologica quanto fisica, è già presente nelle analisi demartiniane:

[...] l'uomo magico è esposto al rischio della labilità nelle sue solitarie peregrinazioni, allorché la solitudine, la stanchezza connessa al lungo peregrinare, la fame e la sete, l'apparizione improvvisa di animali pericolosi, il prodursi di eventi inaspettati ecc., possono mettere a dura prova la resistenza del 'ci sono'. L'anima andrebbe facilmente 'perduta' se attraverso una creazione culturale e utilizzando una tradizione accreditata non fosse possibile risalire la china che si inabissa nell'annientamento della presenza. (De Martino 1997: 82)

Poco oltre, l'antropologo fornisce un esempio di viaggio, sottoposto a tutela da parte di un istituto culturale collettivamente accreditato: quello «psichico dello sciamano ai superi o agli inferi», quello in cui, cioè, «il contenuto della visione [...] è lavorato con temi mitici tradizionali» (De Martino 1997: 82) ${ }^{8}$. Che il tragitto compiuto da Manuele si sovrapponga a un'ideale discesa agli inferi - immagine, questa, usualmente associata a contenuti apocalittici - è già, per il lettore, un'acquisizione testuale, dotata inoltre, per intenzione narratoriale, dei tratti della impersonalità, ovverosia travestita da esperienza universale: «A volte - specie in certe solitudini estreme - nei vivi prende a battere una pulsione disperata, che li stimola a cercare i loro morti non solo nel tempo, ma nello spazio» (Morante 1982: 6). Se diamo per noto l'oggetto della visione, cioè il colloquio con gli spiriti, quale si esplicherà nel racconto, e prendiamo per tradizione culturale la forma stessa del raccontare e, in seconda battuta, l'istanza biografica veicolata dal testo, resterebbe da definire quale motivo mitico funga da garante per il cammino andaluso nel mondo magico, nella «preistoria» ${ }^{9}$ (Morante 1982: 9) di Aracoeli.

${ }^{8}$ In maniera omologa, d'altra parte, si esprime lo stesso Manuele: «[...] nel montare sull'apparecchio ho conosciuto la sensazione che forse prova lo sciamano entrando nel sonno magico. [...] $\mathrm{m}$ 'ha fasciato una sorta di narcosi, che mi isola dai fenomeni ordinari e dalla gente. Gli altri viaggiatori dell'aereo [...] si avviano tutti a destinazioni usuali, calcolate dalla ragione. Io solo salpo verso El Almendral: estrema punta stellare della Genesi» (Morante 1982: 20), dove le nozioni antropologiche di Morante si contaminano con la reminiscenza biblica.

${ }^{9}$ Il rilievo del termine «preistoria» è attestato altresì dalla di poco precedente occorrenza lessicale (Morante 1982: 4), al centro di un campo semantico, costituito dagli emblematici «segreto», «arcano», «tesoro», che contribuisce a qualificarlo nel senso dell'alterità. 
Anche in questo caso, ci sembra che il testo organizzi da sé la propria griglia interpretativa. Il secondo paragrafo del primo capitolo («Sono passati trentasei anni da quando mia madre fu sepolta nel cimitero di Campo Verano [...]. E sono più di trent'anni che non rivedo Roma, dove non penso di tornare mai più», Morante 1982: 6) costituisce, infatti, a dispetto dello stile cronachistico che ne smorza l'impatto, il punto di tangenza tra due formazioni culturali: il mito dell'orfano primordiale e la condizione apocalittica degli ultimi: «di coloro che per ultimi conoscono e nominano con estrema volontà di evocazione e di possesso le realtà dell'universo o del ciclo cosmico prossimo alla fine»l ${ }^{10}$ (Jesi 1992: 10).

La figura dell'orfano sembra dover essere tale poiché in essa si fondono l'esperienza dei terrori dell'uomo solo nel mondo primordiale e la fiducia in una fatale ripetizione: fiducia nella salvezza garantita dall'essere l'orfano una ripetizione del padre, e dal perenne ritornare del tempo. Riferita alla cosmogonia, questa situazione deve necessariamente far coincidere all'immagine del padre assente e più tardi soccorritore quella di un dio. Ma ciascuno, volgendosi verso la propria nascita, contempla e sperimenta una cosmogonia; e se [...] non vi è religione che garantisca la presenza del 'padre nei cieli', l'orfano fisserà i suoi occhi sulla fine del ciclo iniziato con la sua nascita e si sentirà [...] il predestinato a nominare e ad evocare per l'ultima volta gli arredi del proprio universo. (Jesi 1992: 11)

La condizione di Manuele è, dunque, quella dell'orfano «senza i conforti della religione», vittima di un abbandono epocale, storicamente determinato, condannato cioè alla doppia distanza dalla madre e dalla città-utero, la Roma dell'infanzia'11, città babilonesca e apocalittica par excellence, che, subito dopo, con la riportata notizia storica del bombardamento sul cimitero del Verano, ci è offerta quale luogo dalla spazialità devastata dagli orrori bellici. La città diviene, dunque, il simbolo di una maternità problematica, non solo dal punto di vista del narratore, che ne deriva, con la nascita, una «separazione sanguinosa» (Morante 1982: 18), ma anche per la protagonista del racconto biografico, Aracoeli, che vi esperisce l' «atrofizzarsi delle sue possibilità di maternità» (De Martino 2002: 45) a partire dal lutto subito nella persona della neonata Encarnación-Carina, che duplica, a breve distanza, la scomparsa, sottaciuta alla partoriente, del fratello Manuel. A questo punto, nello spazio della cronaca («A quell'ora, io mi trovavo a lezione», Morante 1982: 203), si innesta la circostanza del referto, per cui, nella particolare esperienza della protagonista, la «crisi del cordoglio» entra in contatto col «tema psicopatologico della fine del mondo» ${ }^{12}$ (De Martino 2002: 91):

${ }^{10}$ Così lo studioso si esprime a proposito del simbolismo pascoliano, che abbiamo già riconosciuto come una possibile fonte del particolare simbolismo di Morante.

${ }^{11}$ Un consuntivo dei doppi geografici attivi nel romanzo è offerto in Martínez Garrido (2009: 118-127).

${ }^{12}$ Tale tema sembra permeare inestricabilmente la narrativa degli anni '60. A noi basteranno due esempi tratti dall'autrice, che per più voci è passata quale emula della nostra, 
[...] il cordoglio è appunto il faticoso ridistribuire i significati del proprio mondo in modo da fare a meno della presenza vivente della persona morta, convertendo il suo 'vuoto' in cara memoria. Ma nella misura in cui tale conversione non è ancora compiuta, ha luogo il doloroso vissuto di un "mutamento di significato". (De Martino 2002: 92)

In tale «mutamento di significato», che si traduce, con immaginazioni euforiche o disforiche a seconda che nel malato prevalga l'atteggiamento maniacale o quello malinconico-depressivo, in una perdita di familiarità con il mondo precedentemente abitato, nel senso di un attivo fare e relazionarsi con altri, la crisi del cordoglio si tramuta in crisi della presenza e attiva un «delirio di negazione» (De Martino 2002: 113), ossia di rifiuto della realtà, che colpisce fin la propria persona. A questo proposito, De Martino menziona come significativo il caso di una donna, che rinnega l'universalità del proprio vissuto:

- Come vi chiamate?

- Mi facevo chiamare Celina... Non ho più nome...

- Siete sposata?

- Vivevo con un uomo chiamato Giovanni: lo chiamavo mio marito e non poteva esserlo (la malata è tuttavia sposata legittimamente). Avevamo stabilito di restare insieme ventotto anni, sino alla fine del mondo.

- Avete figli?

ovvero quell'Anna Maria Ortese, che nel 1969 pubblica, nella raccolta omonima, il racconto L'alone grigio (Ortese 2006: 267-275), in cui il senso di una imminente catastrofe naturale coincide significativamente col ritorno dei defunti compianti, tra cui gli amati genitori della narratrice autodiegetica: «Intanto, i fenomeni, intorno, assumevano un'intensità sempre più inquietante, la popolazione era informata, qui e fuori. Stupiva il numero immenso di gente che tornava, e stupiva ancor più il sentimento affettuoso, di normale piacere e gioia, con cui era accolta. Si aggiungeva a questi ritorni, un altro fatto sottilmente terrificante: tutte le partenze erano cessate. I malati e i vecchi non se ne andavano più. Tutte le partenze erano sospese, cessate. Anche perciò i viveri scarseggiavano. Il latte, da un mese introvabile. Ora anche il pane» (Ortese 2006: 269, i corsivi sono dell'autrice). La seconda suggestione arriva dal racconto Casa di bambola, pubblicato in rivista a più riprese dal 1958 (Ortese 2006: 415-432), che avvia un complesso gioco di equivalenze tra lo stato di angoscia planetaria per una prevista esplosione del Sole, il fallimento politico del Partito Comunista sulla scena nazionale e internazione, e la crisi esistenziale e sentimentale della giovane protagonista: «"Splende fino al Canadà l'Aurora Boreale!”. Mio Dio, abbiate pietà della mia debolezza. Perdonatemi se piango mentre leggo questo titolo. So che vi è della speculazione, e della nevrosi anche, in questi fatti, ma essi mi ricordano troppo da vicino una verità irrespingibile: la vita dell'uomo sulla terra si rivela sempre più gratuita, nella sua meccanicità, meno sicura, meno significativa, e il deserto cresce intorno alle opere stupende dell'uomo. [...] E, allora, che cos'è questo deserto, questa brutalità, questo dolore, questo trionfo delle cose impassibili? Che significato ha tutto ciò?» (Ortese 2006: 424). Si consideri come tipico della letteratura apocalittica il topos della desertificazione, che troveremo presente nella stessa Morante. 
- Avevo un figlio che dicevo il figlio di Giovanni... ma non era mio figlio ${ }^{13}$. (De Martino 2002: 115)

Un primo rilievo sul brano citato riconduce al contesto originario della pubblicazione, ossia all'articolo demartiniano, Apocalissi culturali e apocalissi psicopatologiche ${ }^{14}$, apparso sulla rivista Nuovi Argomenti nel 1964, ossia ad un'altezza cronologica in cui Morante è presumibilmente ancora impegnata nel progetto romanzesco di Senza i conforti della religione, poi abbandonato in favore de La Storia, ma il quale, a nostro avviso, si riparte tematicamente e poeticamente in questo come nel successivo Aracoeli, non soltanto per la presenza di un personaggio femminile omonimo ${ }^{15}$. Seppur resta più che valida l'ipotesi della suggestione storico-biografica ${ }^{16}$, col rimando alla sorella della pensatrice spagnola, María Zambrano, entrambe esuli per volontà anti-franchista e risiedenti a Roma fino al 1964, potremmo pensare, altresì, a un rafforzamento qualificativo dell'impressione, esercitata su Morante dalle personalità menzionate, tramite l'accredito scientifico della testimonianza demartiniana, specie se si consideri il dato, nuovamente di carattere biografico, della acuta nevrosi di Araceli Zambrano. Come il caso di Celina, riportato dall'antropologo, si attagli all'esperienza del personaggio Aracoeli, a partire dall'abbandono del tetto coniugale ${ }^{17}$, risulta evidente, infatti, dal passo che De Martino pone a premessa dell'interrogatorio clinico $^{18}$ :

13 Il corsivo è nostro. Si noti, qui, che l'antropologo traduce da studi scelti di area francofona, italianizzando dunque gli appellativi dei pazienti clinici.

${ }^{14}$ Cfr. De Martino (1964: 105-141). Il brano riportato vi compare a p. 134. Ai fini di una corretta ricostruzione della ricezione dell'opera di De Martino da parte di Morante, ci pare interessante un confronto con le occorrenze demartiniane in Pasolini, con particolare riguardo all'articolo citato, che sono oggetto dello studio di Tricomi (2011: 177-195).

${ }_{15}$ A questo proposito, cfr. Di Pascale (1994: 287-302).

${ }^{16} \mathrm{Si}$ veda, per questo aspetto, oltre al già citato intervento di Martínez Garrido, anche il contributo di D'Angeli (2003: 74-75), dove si attesta l'utilizzo morantiano del diminutivo Celina in Senza $i$ conforti della religione, come pure si legge nel breve paragrafo introduttivo alla riedizione del romanzo, apparsa nel 1992.

${ }^{17} \mathrm{Si}$ badi che, proprio a partire dal primo contatto tra Aracoeli e la Donna-cammello, nella cui «quinta» trova accoglienza e sfogo la ninfomania che impania la donna, come conseguenza della malattia e della crisi psicologica, si attesta l'utilizzo nel romanzo del diminutivo «Cielina» (Morante 1982: 253s.), che anzi Manuele riferisce coniato dalla stessa Signora, quasi a marcare, con quella onomastica, l'alterità della natura di Aracoeli in connessione agli sviluppi successivi della sua vicenda esistenziale.

${ }^{18}$ L'importanza del passo precedentemente citato, a nostro avviso, è rimarcata dalla ripresa morantiana, seppur in chiave parodica, del modulo dell'interrogatorio in due puntichiave del romanzo: l'inquisitoria processuale, ma anche esempio di «studio clinico o psichiatrico», che ha al suo centro la figura del narratore «IMPUTATO» (Morante 1982: 113118), e la farsesca inchiesta, con cui si conclude la pseudo-avventura partigiana di Manuele, che vi compare nuovamente nei panni dell'«IMPUTATO» (Morante 1982: 155-158). 
Nel cosiddetto delirio di negazione il vissuto di annullamento esistenziale concerne innanzi tutto la propria personalità morale e intellettuale, per investire poi il proprio corpo, gli altri, il mondo. Non si ha più pensiero, cuore, sentimenti, nome, età; non si ha più stomaco, lingua, cervello, testicoli, sangue, pene; non si ha più parenti, amici; tutto porta il segno della morte, terra, stelle, alberi, stagioni. Nella perdita di ogni possibile progettazione secondo valori intersoggettivi, l'orizzonte del futuro si chiude e il nostalgico paradiso dell'esistenza si riduce alla struggente memoria del passato in cui il mondo c'era ancora, sino al momento in cui il tempo si è rattrappito e il divenire si è fermato. (De Martino 1964: 133-134)

In Aracoeli, il processo di negazione, che culmina come per Celina nel ripudio della condizione muliebre e materna («E invece, io non sono più la tua mamita», Morante 1982: 268 ${ }^{19}$ si esplica a partire dal tipico rifiuto dell'alimentazione ${ }^{20}$, che pone al centro dell'attenzione la disfunzione dell'oralità, quale si esemplificherà con maggior pregnanza nel mutismo o nell'inarticolazione del suono col conseguente assottigliarsi della significanza del messaggio o, per essere più esatti, con 1'accesso a una dimensione "altra", quella della verbalità pre-logica e magica: «Pareva, all'udirla da fuori, un'invasione di cani rabbiosi o di belve [...] un lagno solitario e miserabile, simile a un rantolo; da cui le prorompeva d'un tratto, a volte, uno strido quasi sguaiato, come di uccello notturno [...] frasi spagnole incomprensibili (forse scongiuri)»» ${ }^{21}$ (Morante 1982: 203-206). Lungi dal «non avere

${ }^{19}$ Ma ancora più emblematico è il biglietto di addio lasciato al marito: «ANDATA VIA PARASIEMPE FINITTO IO / NO SONO DIGNA DESER TUA ESPOSA» (Morante 1982: 274, così nell'originale), dove l'arbitrario andare a capo, che separa il pronome di prima persona dal predicativo nominale che segue, appare un'intenzionale scelta grafica dell'autrice, non tanto per rimarcare la condizione pre-grammaticale della scrittura aracoeliana, quanto per congiungere, in posizione forte, l' «IO» al senso di finitudine essenziale.

20 «Non ho più stomaco: non ho mai la sensazione di avere fame. Quando mangio, avverto certo il gusto degli alimenti, ma quando sono in gola non avverto più nulla, è come se cadessero in un buco» (De Martino 2002: 114); «In quelle giornate, il suo stomaco recusava ogni cibo» (Morante 1982: 205).

${ }^{21} \mathrm{Si}$ tratta, qui, a ben guardare, del corrispettivo negativo della lingua materna che Manuele possiede dall'infanzia («Con le sue prime nenie di culla [...] la sua tenera voce di gola, intrisa di saliva [...] mi ricanta all'orecchio quelle sue canzoncine di paese $[\ldots]$ certe piccole voci di affetto e risatine scherzanti [...] gli accenti spagnoli [...] che piovono su di me dalla sua bocca ridarella come un tubare dall'alto», Morante 1982: 13). Le due lingue, per quanto connotate oppositivamente in termini di ricezione emotiva, si situano tuttavia entrambe nella zona di frontiera tra linguaggio verbale e linguaggio pre-verbale. Tale dato comune determina, nel corso del racconto biografico di Manuele, frequenti episodi di sovrapposizione erronea dei dati mnestici, episodi traducibili, a livello narratologico, col complesso sistema romanzesco di analessi e prolessi (a riguardo scrive Rosa: «La crisi della componente narcisistica [...] esplode nella frammentazione del racconto, che altera e corrode l'antica relazione fra io narrante e io narrato, fra tempo del discorso e tempo della storia», Rosa 2006: 297), ma che, nell'ottica alterata di Manuele, diventano sintomi patologici: «E allora d'un tratto mi attraversa una sensazione orribile: quasi che in questo 
nel romanzo un peso semantico autonomo» (Serkowska 2002: 7), dunque, l'episodio della nascita e della prematura morte di Carina, oltre che come indicatore di una variata tipologia testuale, funziona, non tanto da innesco, quanto da acceleratore delle dinamiche di disfacimento psico-fisiologico della madre, avviato già con la gravidanza, e che, dalle parole di Manuele, ottiene significativamente uno stigma di ordine sociale dai toni vagamente esaltatori, nei termini di quell' «anarchia passiva» che è «ritmo naturale, libero e illegale» (Morante 1982: 187) e che, in quanto tale, con la rinuncia a una personalità e a un ruolo pubblici, si configura quale doppio prospettico della «resistenza all'azione» (De Martino 2002: 115) che interverrà di lì a poco. Solo successivamente il nuovo stato di Aracoeli comincia ad acquisire il contrassegno del brutto e del patologico («Apparentemente, s'era imbruttita [...] la vedevo farsi pallida, quasi verde, e sturbarsi, o correre via con le mani alla bocca $[\ldots]$. Trasandata, mal pettinata, sempre in vestaglia e ciabatte $[\ldots]$ sfiancata e torpida», Morante 1982: 197-195).

Il nesso tra le due connotazioni, quella sociale - positiva, ma per approssimazione a un "tipo" - e quella estetica - demolitoria del mito della bellezza materna - è da ricercarsi più oltre, quando, con il risveglio dal sonno chimicamente indottole durante la veglia funebre, Aracoeli appare al figlio nelle vesti di «una sonnambula, o una balorda» (Morante 1982: 204). L'evoluzione semantica subita dal termine "sonnambula» attesta l'emersione di una storia culturale che si apre all'insegna dello scandalo collettivamente condiviso, passa attraverso la diagnosi clinica dell'analisi psicopatologica, ma intanto assurge a istituto di ritualità sociale $^{22}$. Nato in contesto sensista, ma in funzione anti-sensista prima e antipositivista poi, il sonnambulismo sviluppa il proprio mito attorno al corpo simbolizzato $^{23}$ della sonnambula, alternativamente riconosciuta, e stigmatizzata dai poteri forti, come una posseduta, un'estatica, un'alienata o un'isterica ${ }^{24}$, ossia

incomprensibile balbettio lei mi significasse un avvertimento [...] di un male senza nome» (Morante 1982: 13).

${ }^{22}$ «La parola "sonnambulo" ha ormai cambiato di segno in modo radicale: non indica più solo chi cammina nel sonno, ma anche chi sviluppa particolari tecniche psichiche e manifesta capacità straordinarie all'interno di un ruolo socialmente riconosciuto») (Gallini 1983: 37).

23 «Nella sonnambula, il corpo è dunque presente assieme come realtà e come mito, come materia e come simbolo, con una prossimità tra i due livelli - quello del reale e quello dell'immaginato» (Gallini 1983: 34).

${ }^{24}$ Sulla base di tali pregiudiziali, che peraltro sembrano riprodurre il punto di vista socialmente orientato della famiglia paterna di Manuele bambino, ci troviamo evidentemente al confine tra forme di pensiero a-scientifico e risultati di analisi di stampo alienista, sostanzialmente pre-freudiane, per una coerenza cronologica rispetto alla situazione di stallo dei saperi istituzionali in epoca fascista. Pertanto, potremmo prendere in considerazione l'eventualità di un «paradigma psicoanalitico deriso» (a tal proposito, cfr. Serkowska 2002: 17-20), solo postulando l'inapplicabilità dello stesso alle porzioni testuali interessate dalla rievocazione mnestica della vicenda biografica di Aracoeli e avanzando la 
soggetta a una perdurante condizione di debolezza ed emarginazione sociali. L'effetto perturbante legato a tale figura andrà, tuttavia, ricercato proprio nella dimensione di una fisicità concorrente rispetto a quella che, comunemente, ottiene la ratifica della normalità ${ }^{25}$, sia che, a quell'altezza cronologica, si limiti il fenomeno a una patologia del sonno sia che lo si riconduca a più complesse teorie organiciste.

Ciò che, qui, ci preme rilevare è come l'analogia tra lo stato di Aracoeli e quello sonnambolico non sia un mero espediente retorico, ma un motivo testuale consapevole e insistito. Infatti, se è vero che il sonnambulismo «fa esplodere le usuali regole dell'esperienza e della conoscenza per ricostruire le proprie coordinate su un sistema di riferimenti che simmetricamente vi si oppongono» (Gallini 1983: 53), alterando cioè i principi spazio-temporali e di non contraddizione della logica cartesiana, abolendo il criterio di predominio della vista sugli altri sensi, registrando e teatralizzando, infine, quella comunicazione invisibile tra corpo e mente, per cui la sonnambula acquisterebbe la chiaroveggenza del male proprio o altrui, potremmo accertare che quella di Manuele, sulla condotta e le sembianze di Aracoeli, sia una forma di diagnostica per immagini che sottrae l'individuazione del principio materno al paradigma di realtà e la riconduce sotto il segno del mito. Inoltre, la torbida vicenda aracoeliana di erotismo e morte, che culmina nelle scene di onanismo e di seduzione del giovane attendente Daniele, corrisponde a uno scivolamento nella mania euforica, intesa come interruzione e non conclusione del lutto (Lewin 1971: 66). Tanto il sonnambulismo, dunque, quanto la perversione sessuale appaiono quali elementi di «diniego attivo» ${ }^{26}$, meccanismo di difesa dall'angoscia esistenziale.

Quanto detto spiegherebbe già l'avversione della madre per gli occhiali, che deturpano il viso del bambino, nei modi di una istintiva misura di dequalificazione di uno strumento di conoscenza tradizionale e nient'affatto empatico, come la lente, simbolo dell'applicazione sistematica dell'organo della vista, appunto. La reazione di Aracoeli, al momento della prova e dell'acquisto degli occhiali, è attribuita dal narratore, che ripropone invariata l'ottica del bambino Manuele, a una ripulsa del gusto:

'Non gli stanno bene', la udii protestare [...]. Nella sua protesta, impigliata fra la timidezza e la passione, fiatava un'autentica, furente ferocia; e [...] non l'occhialaio soltanto era oggetto della sua rabbia; ma anch'io! [...] Per una violenza - si direbbe -

riserva che il fallimento dell'istanza di autoanalisi in Manuele adulto sia da ascrivere, appunto, a un retaggio dell'educazione introiettata, più che a una effettiva posizione critica.

${ }_{25}$ Secondo Gallini, infatti, «[...] il materiale simbolico espresso dal corpo della sonnambula» ha "carattere di residualità che lo definisce rispetto al modello dominante di una ragione di veglia, dai cui confini è stato bandito» (Gallini 1983: 47).

26 «Il contenuto delle euforie è un diniego diretto di un fatto, viene creduto volentieri e senza riserve, e quindi assomiglia nel modo di funzionamento ai sogni creduti dei bambini» (Lewin 1971: 76-77). 
fuori dalla sua volontà, essa mi scrutava, e i suoi tratti parevano scomporsi, quasi invecchiati dalla sorpresa e dalla delusione, come alla scoperta di un tradimento. Difatti (io credo) per la prima volta nella nostra vita, essa mi vedeva brutto. (Morante 1982: 174)

La spiegazione della condotta non episodica di Aracoeli nei confronti del figlio occhialuto potrebbe, tuttavia, più significativamente essere motivata, se si prestasse attenzione a quanto affermato da Manuele subito dopo, a proposito della risposta, anch'essa tutta emotiva, della madre alla preparazione scolastica del bambino, dalla quale si evince la possibilità, per la donna, di accesso a modalità di conoscenza alternative. In questo senso, non ci sembra un caso che la menzione degli occhiali anticipi una problematica di tipo gnoseologico ed epistemologico.

I miei successi scolastici [...] la intimidivano. [...] la mia istruzione già superava la sua. [...] dopo la grande conquista dell'alfabeto e dell'italiano, il suo intelletto oramai toccava una frontiera [...] essa lo sentiva senza saperlo, attraverso una sua intelligenza quasi fisica, nascosta persino a lei stessa [...]. La sua era un'intelligenza diversa dalla nostra: era una sostanza ombrosa, imperscrutabile e segreta, che scorreva in tutto il suo corpo, quale un'infinita memoria carnale [...]. Essa la rendeva capace [...] di avvertire, negli spazi e nei tempi, presenze, movimenti e meteore negate alla nostra cognizione [...]. L'intelligenza misteriosa, che non aveva stanza nel suo pensiero, era una pellegrina incognita dentro di lei. (Morante 1982: 177)

Morante ricorre alla stessa radice lessicale («timidezza», «intimidivano») per definire le ragioni di una estraneità patita da Aracoeli, benché a lei connaturata, e che, mentre la penalizza rispetto alla convenzionalità del conoscere, le schiude le porte di un sapere unionale, che fa di corpo e mente un complesso indistinto. Prova ne sia l'«evento oscuro», inspiegabile per il bambino Manuele, ma che per il narratore costituisce ormai, a riguardarlo nella memoria, «il segnale di un incendio» (Morante 1982: 213).

Un doppio rivolo di sangue continuava intanto a colarle giù lungo le gambe, allargandosi per terra in una pozza che lei spiava perplessa. Dondolò appena il capo, emettendo un brevissimo lamento infantile, quasi tenero. E di colpo si accucciò sul pavimento, come un animale sfiaccato da un troppo peso. [...] Lungo il breve percorso dal corridoio al letto, il tappeto s'era macchiato di sangue, mentre il volto di Aracoeli, di un pallore quasi bluastro, manteneva una sua strana espressione riflessiva. Essa non aveva perduto i sensi, anzi pareva riprendersi un poco, e [...] bisbigliò molto seria, come le dovesse una spiegazione:

«Il mio male è qua

- e qua».

E si toccò prima l'addome e poi la fronte. (Morante 1982: 214) 
Oltre a veicolare 1'idea fondamentale del male come contagio ${ }^{27}$, come metastasi o rizoma, la sequenza rimarca la capacità di autocoscienza della protagonista (con la «sua strana espressione riflessiva») mostrandosi, del resto, a un livello macrotestuale, quale compiuta sinossi di alcuni luoghi cardine della poetica morantiana con il richiamo all'animalità in combinazione con una sopravvivenza antropologica infantile. Di qui, è inevitabile l'approdo alla funzione morantiana di un senso del primitivo, alla luce del quale anche il binomio «addome»-«fronte» subisce una significativa ridefinizione nell'ambito delle tradizioni procreative della cosiddetta «psicologia primitiva». Stando agli studi etnologici condotti da Malinowski, infatti, alcune società primitive di carattere matrilineare sarebbero affatto estranee alla concezione delle dinamiche di fecondazione, e persino delle rispettive particolarità anatomiche, restando ancorate a un immaginario «motivo dell'inserimento attraverso la testa, motivo al quale [...] di solito [...] viene associata l'idea di un afflusso di sangue, prima nella testa, e poi nell'addome»» (Malinowski 1990: 89). Il male di Aracoeli, che dall'addome risale alla testa, dopo la perdita del flusso vitale, costituisce l'inversione di un tale processo procreativo e segnala il regresso della funzione materna nel personaggio. Che poi la maternità, nella concezione morantiana, debba assumere una simile qualifica asessuale e pregenitale ci pare ribadito dalla scelta onomastica, che assegna alla neonata Carina il marchio dell'incarnazione o re-incarnazione, se restiamo nel solco della psicologia primitiva, come da plurime occorrenze testuali. Se Manuele definisce, infatti, la materna come una «aspettazione inconsapevole del seme» (Morante 1982: 103), siamo di fronte a un tipo di mentalità - quella di Aracoeli -, per la quale il «fluido seminale [...] non è dotato di alcun valore generativo» (Malinowski 1990: 82), mentre, come dimostrerebbe lo stesso sogno in cui Sant'Anna offre la maternità in «regalo» ${ }^{28}$, il concepimento è il segno evidente dell' «intervento attivo di un altro

${ }^{27}$ Notevole il ricorso, nel romanzo, a scelte lessicali riconducenti all'ambito epidemiale: basti citare il «focolaio di morbi» (Morante 1982: 216), la «lebbra» (Morante 1982: 235), la «pestilenza» e la «concrezione pullulante» (Morante 1982: 237), il «teatro infetto» (Morante 1982: 239), ecc.

${ }^{28}$ «Quella stessa notte, tu sognasti che t'era cascato un dente, e tu lo deponevi in cima al tetto, in offerta a Sant'Anna, la nonna di Dio: la quale, a volte, in cambio di tali offerte, per consolazione lascia un regalo. Di lì a poco, da sopra il tetto si udiva uno stridolío, come di un grillo. E tu, accorsa, in cambio del dente ci trovavi una fava secca. Ma dentro a questa fava, poi, ci si trovava una niña, di misura minima, però intera e completa, che già muoveva gli occhi. Era vestita da reginella, e in testa, per corona, portava un anellino d'oro. / Per te, un simile sogno valeva quanto un avviso ufficiale: una specie di telegramma, spedito al tuo indirizzo dalle supreme Autorità del Cosmo» (Morante 1982: 104). Il sogno in esame ci interessa altresì per il dettaglio del dente caduto che rimanda alla determinazione che Aracoeli darà alla morte del fratello Manuel in risposta alle domande del figlio («Manuel ha perso i denti», Morante 1982: 231), per cui si evince non soltanto come la morte di Manuel anticipi quella di Carina, nella misura in cui la possibilità della nascita è associata simbolicamente alla caduta del dente, che ritorna poi come immagine luttuosa, ma anche che la scomparsa dello stesso risulti necessaria per la venuta al mondo della bambina in termini 
spirito», che recherebbe alla puerpera l'anima di un defunto reincarnata nel corpo di un nascituro:

Ci sono degli indigeni, i quali immaginano che gli spiriti più anziani portino il piccolo in qualche sorta di ricettacolo - un cesto di fibre di cocco intrecciate o un piatto di legno oppure semplicemente nelle braccia. [...] L'essenziale di questa versione tuttavia sta nel fatto che dietro il bambino, esiste il controllo attivo di un altro spirito. [...] Un tratto tipico del comportamento di questo spirito controllore è che di solito appare alla donna che sta per diventare incinta. (Malinowski 1990: 90)

Se il sogno di Aracoeli manifesta compiutamente il fondo di sincretismo pertinente a una forma di religiosità popolare, la quale combina permanenze antropologiche di antico retaggio culturale (il motivo del sogno rivelatore quanto quello del contenitore per il bambino) con l'ingenuo immaginario cattolico di una depauperata annunciazione, sono poi le affermazioni del narratore a suffragare l'esistenza di un tipo di credenza nella metempsicosi:

[...] questo percorso mi è già noto. Chi sa in quale altra mia biografia, già una volta mi sono avviato in questa direzione [...]. Può darsi che quell'altra mia biografia sia soltanto immaginaria, un riflesso effimero di questa; ma è anche possibile che questa odierna, invece, sia solo un riflesso dell'altra: la vera. Si dànno, nel campo della luce, simili giochi di specchi. (Morante 1982: 139)

L'utilizzo del termine «biografia» come sinonimo di un più prevedibile "vita" sembra farci scivolare sul terreno, già praticato in Morante, dell'alibi, del doppio letterario, per cui la presenza dell'io narrante (e scrivente, al limite) prenderebbe sostanza dalla proiezione in un io narrato. Un secondo passo del romanzo autorizza tale lettura da un lato, rinforzando, dall'altro, con l'emersione metamorfica dell'animale totemico, la spia del primitivismo:

Non ero addormentato, ma nemmeno sveglio del tutto; quando sul vetro della finestra, incominciò a svolgersi, come viva, una piccola scena in movimento, simile nelle tinte alle miniature, o alle vetrate delle cattedrali. E il protagonista ne ero io, sebbene assunto in una forma altra dalla mia forma ordinaria. Ero una fanciulla indiana bellissima (forse una danzatrice sacra?) [...]. Ma questa era soltanto una prima metamorfosi: poiché subito i fiori, a loro volta, si trasmutavano in lunghe piume variopinte, per comporsi definitivamente a formare un uccello fantastico, di tutti i colori dell'iride. Una fenice!

di testimonio spirituale e sempre alla luce delle testimonianze antropologiche sulle credenze primitive, per cui «la vita nuova [...] incomincia con la morte» (Malinowski 1990: 85). Nel caso della precedente nascita di Manuele (a cui il sogno si riferisce), evidentemente, la morte necessaria è quella della stessa Aracoeli, che abbandona la terra d'origine e segue lo sposo in una sorta di viaggio iniziatico, che la introduce a una nuova esistenza, sotto il segno peraltro di una ben distinta connotazione sociale. 
[...] Rammento che non provai nessuna sorpresa a riconoscermi nella fanciulla indiana trasformata in una fenice. (Morante 1982: 140-141)

L'immagine della fenice è qui, analogamente a quella del gatto Alvaro ${ }^{29}$ in Menzogna e sortilegio, ipostasi della stessa operazione scrittoria, della finzione appunto, come si desume del resto dal contesto lessicale che rimanda, nella visione dirompente delle cattedrali e dei fiori estivi, al Diario 1938, dove appaiono in nuce le prime dichiarazioni morantiane di poetica. Ma la fenice, in quanto simbolo metamorfico $^{30}$, e pur se in un momento di visionarietà maniacale alterata dall'uso di stupefacenti da parte di Manuele, è evidentemente anche il segno sotto il quale si pone il narratore per garantire al lettore la propria condizione di alterità, posta dalla (ri-)nascita, appunto, e perciò connessa col vincolo della genitorialità rinnegata dall'orfano. Scopriamo allora che, nei meccanismi associativi di Manuele, stimolati dalla narcosi autoindotta o dal sonno naturale, un altro personaggio trova la propria configurazione per entro il contesto animale, rimarcando pertanto la parentela col narratore e sottraendo quest'ultimo alla sfera di influenza simbolica della figura paterna, la quale, anche se abbrutita nel finale, non assurge mai al regno della bestialità favolosa. Tale personaggio è proprio il fratello andaluso di Aracoeli, quel Manuel che si manifesta, in sogno, «piccolo, circa della mia misura; e inoltre, invece che un cavallo, cavalcava un toro. [...] un essere gigantesco, nero» (Morante 1982: 198), manifesta immagine ctonia, laddove già il cavallo risulta in connessione col rito funebre primitivo e il toro una mera variante mitografica ${ }^{31}$. Di più, «il toro

29 «Cases ha giustamente osservato che l'immagine conclusiva del gatto Alvaro suggerisce un'idea di letteratura in funzione di autorisarcimento consolatorio [...]. Ma [...] in tanto è metafora della fantasia ispiratrice quanto più ne esemplifica le tensioni conturbanti» (Rosa 2006: 24).

${ }^{30}$ Sin dalla testimonianza di Erodoto, tuttavia, attorno al mito della fenice si diffonde una duplice tradizione: essa non guida soltanto l'identificazione col defunto che, in contesto egizio, al modo di una divinità solare, è soggetto a un periodico ritorno, ma anche quella che compare alla morte del padre, che trasporta altresì il cadavere paterno al luogo di sepoltura. In quanto tale, la fenice si riconnette al mito dell'orfano, che abbiamo già visto sovrapposto al personaggio di Manuele. Altre fonti associano il leggendario uccello tanto al contesto orfico-pitagorico della metempsicosi, a cui peraltro abbiamo alluso, quanto a quello apocalittico di decadenza e rigenerazione cosmologica. Per ulteriori indicazioni, si rimanda Zambon/Grossato (2004).

${ }^{31} \mathrm{Ma}$, vorremmo anche aggiungere, variante localistica, con riferimento al contesto andaluso, cui si intesse un'ulteriore trama di rimandi storico-biografici. Alludiamo, qui, alla figura del poeta spagnolo Miguel Hernández, già incastonato nell'ipertesto morantiano, in quanto sua l'epigrafe finale de La Storia, quasi come un rimando allusivo al romanzo "spagnolo" ancora da compiere. Ebbene, il poeta, collaboratore negli anni '30 di José María de Cossío per una nota enciclopedia taurina, prima della partecipazione attiva alla Guerra Civile e del lungo definitivo imprigionamento, è ricordato da Morante come poeta del lutto, se stiamo proprio ai versi citati ne La Storia («Muerto niño, muerto mío, / Nadie nos siente en la tierra / donde haces caliente el frío», cfr. Morante 1974: 651), tratti da quel Cancionero 
riveste lo stesso ruolo immaginario del cavallo. [...] È sempre un'angoscia a motivare l'uno e l'altro, un'angoscia specialmente connessa a ogni tipo di mutamento, $[\ldots]$ sovradeterminata da tutti i pericoli incombenti: la morte, la guerra, l'inondazione, la fuga degli astri e dei giorni» (Durand 2009: 88-90).

La riconosciuta somiglianza («della mia misura») ${ }^{32}$ con lo zio materno è, a nostro avviso, da ricollegare, contestualmente alle regole di parentela, alla situazione antropologica precedentemente tracciata, e in base alla quale «la posizione sociale si trasmette secondo la linea di discendenza materna da un uomo ai figli della sorella di lui» (Malinowski 1990: 74), al punto che quella di

[...] "padre" è una definizione puramente sociale: è l'uomo sposato alla madre, che vive in una stessa casa con lei e che forma parte della famiglia. [...] descritto dagli indigeni quale $[\ldots]$ uno "straniero" $\mathrm{o}$, in modo ancora più corretto, un "outsider". [...] la parola "padre" $[\ldots]$ non va presa nelle diverse implicazioni legali, morali e biologiche che ha

y romancero de ausencias, che è riconosciuto come l'opera più matura e icasticamente drammatica di Hernández, opera imperniata sul tema dell'assenza della donna amata, del compianto sul figlio Manuel (si badi alla coincidenza onomastica col fanciullo andaluso) prematuramente morto e del distacco dovuto all'esperienza carceraria: diario intimo, dunque, ma intanto opera di meditazione sulle temperie della storia, che non poteva lasciare indifferente la lettrice Morante. Qui, vogliamo citare, per l'importante ricorrenza simbolica, i versi giovanili de El rayo que no cesa nel componimento Como el toro he nacido para el luto: «Como el toro he nacido para el luto / y el dolor, como el toro estoy marcado / por un hierro infernal en el costado / y por varón en la ingle con un fruto». A questo proposito, si confronti Hernández (1970: 16). Da questa, o dalla prima edizione, riteniamo del resto abbia attinto la stessa Morante, proprio in virtù dell'epigrafe de La Storia, la quale, come il volume di Puccini, riporta la lezione «Muerto niño, muerto mío», successivamente sostituita, nelle più accreditate ricostruzioni filologiche, dalla variante «Muerto mío, muerto mío», di cui evidentemente Morante è all'oscuro all'altezza della stesura del romanzo che precede Aracoeli.

${ }^{32} \mathrm{Si}$ veda anche, al riguardo, la considerazione di Aracoeli stessa, per cui la donna «non si saziava di riscontrare in ogni mio tratto questa somiglianza, acclamandola con sorpresa sempre nuova, come se ogni volta tornasse a scoprirla. Da piccolo, essa attestava, il suo hermanito aveva un viso gemello al mio, da confonderci l'uno con l'altro; e davvero io finivo, a momenti, per confondere l'altro in me stesso, tanto più che il caso ci accoppiava anche nel nome. Fra me e lui, secondo Aracoeli, unica differenza era il colore degli occhi, perché lui li aveva negri» (Morante 1982: 121), laddove il contrasto cromatico, accentuato dalla scelta aggettivale non facilior, ha una palese funzione simbolica, ricollocando il fratello sotto il cielo della morte. Un ulteriore segnale di contiguità è poi dato dalla scena onirica dell'incontro agonistico-erotico tra Manuele e Manuel, il quale, però, sul finire del sogno, sembra ibridarsi in una perturbante femminilità (col sesso "simile a una rosa», stereotipo attribuito con prevalenza alla sfera della sessualità femminile, e l'analogia freudiana tra organo genitale e seno materno), ambiguarsi cioè in un equivoco confronto con Aracoeli, per cui il sogno si carica altresì di una vaga sfumatura incestuosa (Morante 1982: 109-110): ipotesi, questa, che non ci sentiremmo di scartare, anche a voler tener conto della sessualizzazione del simbolo teriomorfico del toro/Manuel. Si rimanda a Jung (1965). 
per noi $[\ldots]$. Il bambino viene a sapere che lui non è dello stesso clan [...], che la sua appellazione totemica è diversa. (Malinowski 1990: 75-77)

\section{Mentre:}

All'orizzonte appare un altro uomo [il fratello della madre...] in un altro villaggio. Il bambino impara $[. .$.$] che egli ha qui la sua proprietà e i suoi altri diritti di cittadinanza;$ che le sue prospettive future stanno qui, che qui risiedono i suoi naturali alleati e associati. [...] nel villaggio che deve chiamare "suo proprio", dove vive il fratello di sua madre, suo padre risulta uno straniero [...] che il fratello della madre assume su di lui un'autorità che, grado a grado, aumenta. (Malinowski 1990: 77)

La persistenza antropologica sembrerebbe attestata, nel romanzo, dal rapporto reale tra Manuele e il padre e da quello virtuale col giovane zio. Se quest'ultimo è definito, infatti, sin dalle prime battute, «il mio EROE» (Morante 1982: 5) e più oltre, stavolta da Aracoeli, «il Gran Condottiero e l'Eroe valente», con un sistema di annominazione fantastica che richiama quello ben più insistito di Menzogna $e$ sortilegio, e intanto sottolinea una uniformità nell'immaginario e identità di stato del pensiero (e del linguaggio) tra madre e figlio, figure non ancora scempiate, il padre, invece, è avvertito come una figura vacante («Allora, per quanto ne sapevo, io non avevo nessun padre. Né concepivo, del resto, che i padri sulla terra fossero necessari e inevitabili», Morante 1982: 120; «Io invece non sono mai stato figlio di un padre», Morante 1982: 183), unita al figlio soltanto in virtù del precedente legame coniugale contratto con la madre, e quindi solo nell'ottica del matrimonio («Fra me e lui, corse subito - in luogo dell'affetto carnale - una silenziosa concordanza: forse anche in virtù della nostra comune passione per Aracoeli», Morante 1982: 134; «[...] da me in lui si onorava lo sposo di Aracoeli, non certo il padre della mia carne», Morante 1982: 183). La riconferma arriva, nell'immediato dopoguerra, dalla visita a Roma che l'adolescente compie alla ricerca di un contatto coi superstiti della propria famiglia. Qui, l'incontro col padre gli suscita, prima di un pianto liberatorio, una sensazione di vissuto apocalittico, animata da immagini disforiche e derealizzanti:

Fuori, la strada moltiplicava, ai miei occhi, il suo movimento di ruote, e piedi, e frotte di ragazzini, e merci, e donne, e divise militari. Il sole era ancora alto nel cielo aperto, ma là d'un tratto delle punte scintillanti mi trafissero le pupille, finché mi parve di vagolare in un sottopassaggio e che gli azzurri ariosi, e la stella diurna, non fossero splendori naturali, ma barbagli elettrici pubblicitari. Anche i passanti, sotto quella strana meteora, mi si deformarono in brutte bambole di carta, o sagome animate da un artificio ${ }^{33}$. (Morante 1982: 325)

${ }^{33}$ Ma si veda già: «Io, se fisso il cielo stellato fino in fondo, lo vedo tutto una fornace nera, che schizza braci e faville; e dove tutte le energie da noi spese nella veglia e nel sonno continuano a bruciare, senza mai consumarsi. Là, dentro quella fornace planetaria, si sconta la nostra vita. È qua, dalle nostre vite, che l'intero LÀ succhia tutta l'energia per i suoi moti. 
Il sentimento di automatismo delle persone, l'artificialità delle cose, l'irrealtà della visione, ovvero lo stravolgimento del proprio sistema percettivo, tutto ciò viene motivato dall'aggallare improvviso di un imprevisto conato d'“amore" per il padre. Ma, a ben guardare, non è forse questa la tendenza narcisistica dell'orfano che si vede riflesso, come da uno specchio, in un altro orfano (e perché vedovo inconsolabile e perché colpito nella propria fede monarchica)? Non assistiamo, dunque, alla reduplicazione del mito degli ultimi, che lanciano uno sguardo di riconoscimento a un reale costretto all'imminente (o immanente) catastrofe? In questa prospettiva, inoltre, l'epilogo del romanzo, con la morte paterna e la presa di coscienza di un bisogno mai estinto di affettività, tende a giustificarne l'avvio, ovvero il motivo del viaggio nel territorio materno, che nel frattempo abbiamo scoperto essere, antropologicamente, la vera patria, il vero «villaggio», di Manuele, aggiunge alla connotazione autobiografica e a quella biografica, da noi inizialmente riscontrate, anche il carattere tipologico del «romanzo terapeutico» (Hillman 1984: 16), in base al quale «il focus del nostro interesse si sposta da un soggetto rivelato a un oggetto esibito, dallo studio del personaggio all'analisi del personaggio e, mediante il personaggio, a una dimostrazione delle mire tendenziose dell'autore» (Hillman 1984: 7). Se Morante, in conclusione, fa sì che la «storia clinica» di Aracoeli, incentrata sull'esperienza dell'apocalisse nel proprio vissuto psicoorganico, sia riassorbita all'interno del «romanzo terapeutico» di Manuele, che invece coltiva, ovvero culturalizza, nei modi della tradizione («il Sabato della paga finale»), ma senza interiorizzarlo, il mito stesso dell'apocalisse, è intanto, come si è detto all'inizio, per ricostruire scientificamente una tassonomia dei valori mitologici, che strutturano l'umano, relativizzandone all'occorrenza il portato. E, infatti, «il fondamento poetico della mente ci dice che la logica selettiva che opera nelle trame delle nostre vite è la logica del mythos, è mitologia» (Hillman 1984: 13). La storia di una crisi diviene, col contributo narrativo di Manuele, una storia «immaginaria» ${ }^{34}$ nel senso «dell'autonomia della memoria dalla storia e della storia dalla memoria» (Hillman 1984: 52) e, in quanto immaginaria, cioè finzionale, essa assurge a cura. Pacifica Aracoeli («"Ma [...] non c'è niente da capire”. La sento che manda un riso, tenero. E questo è l'addio», Morante 1982: 308). Tuttavia, entro l'asse Morante-De Martino, affinché la terapia risulti completa, essa necessita altresì di un ripristino della funzionalità della storia, dell'agire concretamente orientato sul piano degli eventi, laddove invece si consuma inesorabilmente lo scacco di Manuele.

E allora, io vorrei che venisse il Sabato della paga finale: dove l'intero firmamento si spegne» (Morante 1982: 292).

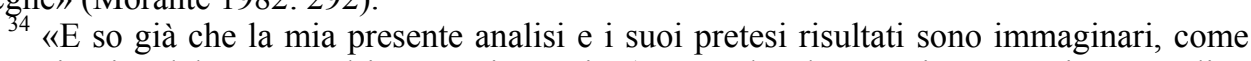
immaginaria, del resto, è l'intera mia storia (e tutte le altre storie, o Storie, mortali o immortali)» (Morante 1982: 327). 


\section{RIFERIMENTI BIBLIOGRAFICI}

BAZZOCCHI, Marco Antonio (2009) «Aracoeli: il personaggio che si annulla», in: Id., Personaggio e romanzo nel Novecento italiano, Milano, Mondadori, pp. 144-157.

D'ANGELI, Concetta (2003): Leggere Elsa Morante, Roma, Carocci.

DE MARTINO, Ernesto (1964): «Apocalissi culturali e apocalissi psicopatologiche», Nuovi Argomenti 69-71, pp. 105-141.

DE MARTINO, Ernesto (1997): Il mondo magico, Torino, Bollati Boringhieri.

DE MARTINO, Ernesto (2002): La fine del mondo, Torino, Einaudi.

Di PASCALE, Anna Maria (1994): «Senza i conforti di alcuna religione», Studi novecenteschi XXI/47-48, pp. 49-65.

DURAND, Gilbert (2009): Le strutture antropologiche dell'immaginario. Introduzione all 'archetipologia generale, Bari, Dedalo.

GALLINI, Clara (1983): La sonnambula meravigliosa. Magnetismo e ipnotismo nell'Ottocento italiano, Milano, Feltrinelli.

HERNÁNDEZ, Miguel (1970): Poesie, Milano, Feltrinelli.

HiLlman, James (1984): Le storie che curano, Milano, Cortina Editore.

JeSI, Furio (1989): Mito, Milano, Mondadori.

JESI, Furio (1992): Letteratura e mito, Torino, Einaudi.

JUNG, Carl G. (1965): La libido: simboli e trasformazioni, Torino, Bollati Boringhieri.

KERMODE, Frank (2004): Il senso della fine. Studi sulla teoria del romanzo, Milano, Sansoni.

Koselleck, Reinhart (2009): Il vocabolario della modernità. Progresso, crisi, utopia e altre storie di concetti, Bologna, il Mulino.

LEWIN, Bertram (1971): Psicoanalisi dell'euforia, Bologna, Guaraldi.

MALINOWSKI, Bronislaw (1990): Il padre nella psicologia primitiva, Milano, Rizzoli.

MARTíNEZ GARRIDO, Elisa (2009): «Between Italy and Spain: The Tragedy of History and the Salvific Power of Love in Elsa Morante and María Zambrano», in Manuele Gragnolati / Sara Fortuna (eds), The Power of Disturbance: Elsa Morante's Aracoeli, Londra, Maney Publishing, p. 118-128.

MoRANTE, Elsa (1974): La Storia, Torino, Einaudi.

MORANTE, Elsa (1982): Aracoeli, Torino, Einaudi.

ORTESE, Anna Maria (2006): Angelici dolori e altri racconti, Milano, Adelphi.

PAVESE, Cesare; DE MARTINO, Ernesto (1991): La collana viola. Lettere 1945-1950, Torino, Bollati Boringhieri.

PISCHEDDA, Bruno (2004): La grande sera del mondo. Romanzi apocalittici nell'Italia del benessere, Torino, Aragno.

RosA, Giovanna (2006): Cattedrali di carta, Milano, il Saggiatore.

SCHOLEM, Gershom (1993): Le grandi correnti della mistica ebraica, Torino, Einaudi.

SERKOWSKA, Hanna (2002): «Percorsi androgini. Aracoeli: il romanzo definitivo di Elsa Morante», Il lettore di provincia 115, pp. 3-27. 
TRICOMI, Antonio (2011): «Il '68: un'apocalisse culturale», in Id., In corso d'opera. Scritti su Pasolini, Milano, Transeuropa.

ZAMBON, Francesca / GROSSATO, Alessandro (a cura di) (2004): Il mito della fenice in oriente e in occidente, Venezia, Marsilio. 